\title{
Estudio observacional del manejo de la hipercolesterolemia en ancianos entre 65 y 75 años
}

\author{
A. Ruiz García, J. E.Villares Rodríguez, B. Herreros Tabernero*, \\ J. C. Hermosa Hernán**, G. del Pozo Sosa***, F. J. Gordillo López**** \\ Médicos de Familia. Atención Primaria Área 10 INSALUD. \\ Unidad de Lípidos y Prevención Cardiovascular Área 10 A. P. \\ EAP Ciudades. Getafe. *EAP Pinto. **EAP Sector III. Getafe. \\ ***EAP J uan de la Cierva. Getafe. ****EAP Pintores. Parla. Madrid
}

\section{Cross-sectional study: dyslipidaemia management in population between 65 and 75 years old}

\section{RESUMEN}

Fundamento: dada la variabilidad del manejo de la hipercolesterolemia en el anciano por los Médicos de Atención Primaria (MAPs), se precisa una evaluación inicial.

Objetivo: describir el manejo de la hipercoleste rolemia en ancianos entre 65 y 75 años por los mé dicos de familia.

Diseño: estudio observacional transversal.

Material y método: de 16 Equipos de Atención Primaria (EAPS) del Área 10 INSALUD, con 125 MAPs, se eligieron a 4 EAPs (25\%) con 38 MAPs, mediante muestreo bietápico por conglomerados, $y$ de los 841 pacientes elegibles, se seleccionaron 551 pacientes mediante muestreo aleatorio simple. Se analizó la existencia de factores de riesgo car diovasculares (FRCIs), cardiopatía isquémica o enfermedad vascular arterioesclerótica (CI/EVA), perfil lipídico, y la adecuación del manejo de la hipercolesterolemia a las recomendaciones del National Cholesterol Education Program (NCEP II).

Resultados: utilizando la prueba Chi cuadrado, se detectó que el 71,87\% (IC95\%: 67,88; 75,55) de los pacientes tenían $\geq 2$ FRCIs además de hiper colesterolemia, o CI/EVA. Se trató la hipercoleste rolemia con dieta exclusivamente en el $64,25 \%$ (IC95\%: 60,07; 68,23), con estatinas un 20,87\% (IC95\%: 17,60; 24,56), con fibratos un $12,16 \%$ (IC95\%: 9,61; 15,25), y con resinas un $2,36 \%$ (IC95\%: 1,32; 4,11). La hipercolesterolemia se manejaba correctamente en el 47,91\% (IC95\%: 43,68; 52,71), disminuía al 41,05\% (IC95\%: 35,33; 47,02) cuando el paciente tenía $\geq 2$ FRCIs, y al 36,94\% (IC95\%: 28,12; 46,67) cuando padecía CI/EVA.

\section{ABSTRACT}

Design: cross-sectional study.
Background: due to variability of dyslipidaemia management by Primary Health Care physicians in population between 65 and 75 years old, is neces sary a baseline evaluation.

Objective: to describe the dyslipidaemia mana gement by family physicians in population between 65 and 75 years old.

Material and method: from 841 patients betwe en 65 and 75 years old with dyslipidaemia, and 16 Primary Health Care centers with 125 Primary Health Care physicians of Area 10 (National Insti tute of Health of Spain), were selected 551 patients and 38 Primary Health Care physicians by alea tory sampling in two stages. Mayor atherosclerotic risk factors (ARFs), coronary heart or atheros clerotic vascular diseases (CH/AVD), lipid profile, and dyslipidaemia management by physicians in accordance with National Cholesterol Education Program (NCEP II) were assessing.

Results: using Chi square test, the 71.87 percent (CI95\%: 37.88; 75.55) of patients had got $\geq 2$ ARFs in addition dyslipidaemia, or suffered CH/AVD. Dyslipidaemia was managed with only diet in the 64.25 percent (CI95\%: 60.07; 68.23), with HMG-CoA-reductase inhibitors in the 20.87 percent (CI95\%: 17.60; 24.56), with fibric-acid de rivates in the 12.16 percent $(C 195 \%: 9.61 ; 15.25)$, and with bile acid sequestrants in the 2.36 percent (CI95\%: 1.32; 4.11). The rate of the accurate dys lipidaemia management in this population was 47.91 percent (CI95\%: 43,68; 52.71), decreased to 41.05 percent (CI95\%: 35.33; 47.02) if patients had got $\geq 2 \mathrm{ARFs}$, and to 36.94 percent (CI95\%: $28.12 ; 46.67)$ if they had got CH/AVD. 
Conclusiones: el manejo de la hipercolestero lemia de la población entre 65 y 75 años no se adecua a la NCEP II en el 52,09\%, empeorando al 59,95\% si el paciente tiene $\geq 2$ FRCIs, y al 63,06\% si padece CI/EVA. Es necesario investigar las razones que influyen en este problema.

Palabras clave: Médicos de Familia. Hiper colesterolemia. Ancianos.
Conclusions: dyslipidaemia management in population between 65 and 75 years old isn't in accordance with NCEP II in the 52.09 percent, de creasing to 59.95 percent if patient has got $\geq 2$ $A R F s$, and to 63.06 percent if patient suffers $C H / A V D$. It is necessary to research what causes have influenced in this question.

Key words: Family physicians. Hyperlipidae mia. Elderly.

\section{INTRODUCCIÓN}

La hipercolesterolemia es un factor de riesgo principal de cardiopatía isquémica (CI) siendo una de las primeras causas de mortalidad. Los estudios epidemiológicos y ensayos clínicos ${ }^{1-5}$ han demostrado que el tratamiento reductor del colesterol reduce la incidencia y mortalidad por CI, y en prevención secundaria también la mortalidad total. La prevalencia $^{6}$ de dislipemia en la población adulta es del $20 \%$, y ésta prácticamente se duplica a partir de los 50 años. La importancia del problema no sólo deriva de esta magnitud. Una adecuada indicación terapéutica consigue disminuir la incidencia de patología cardiovascular y sus tasas de mortalidad.

La Organización Mundial de la Salud ${ }^{7}$ establece como concentración máxima deseable en la población general el valor de $200 \mathrm{mg} / \mathrm{dl}(5,16 \mathrm{mmol} / \mathrm{l})$ de colesterolemia. Las indicaciones actuales ${ }^{8}$ para el tratamiento de la hipercolesterolemia se centran en la existencia de factores mayores de riesgo para cardiopatía isquémica (FRCIs), dirigiendo el mayor esfuerzo en la prevención secundaria, con el objetivo de reducir la concentración plasmática de colesterol LDL hasta valores de $100 \mathrm{mg} / \mathrm{dl}$ (2,6 $\mathrm{mmol} / \mathrm{l})$ en pacientes con $\mathrm{CI} \mathrm{u}$ otras formas de enfermedad vascular arterioesclerótica (EVA).

Existe entre los médicos ${ }^{9-12}$ cierto desconcierto en cuanto al manejo hipolipemiante en ancianos con hipercolesterolemia en presencia o no de CI/EVA, o con FRCIs, bien porque no conocen adecuadamente los criterios que se deben utilizar o por otras causas no científicas. Existen unos criterios $^{13-23}$ aceptados por diversos Consensos y grupos de expertos para el uso de agentes hipolipemiantes en esta población, que vienen determinados por las cifras de colesterol sérico y otros factores individuales. Estas recomendaciones difieren algo en los niveles de colesterol total, colesterol-LDL, y colesterol-HDL, en función del riesgo coronario. Sin embargo, son prácticamente idénticos los ni- veles exigidos para recomendar tratamiento farmacológico ante los pacientes con CI/EVA o ante la presencia de dos o más FRCIs, y éstas se indican claramente en las recomendaciones del Natio nal Cholesterol Education Program (NCEP II) ${ }^{18}$.

En el Área 10 INSALUD (Madrid) existe desde hace varios años un interés especial en el Uso Racional del Medicamento. Un objetivo prioritario es mejorar la relación coste-efectividad de la prescripción de los Médicos de Atención Primaria (MAPs). Esto se implementa con un estrecho seguimiento de indicadores de prescripción farmacéutica a través de una supervisión mensual del ajuste presupuestario a los facultativos, informando de las diferencias entre el presupuesto farmacéutico asignado $\mathrm{y}$ el gasto acumulado ${ }^{24}$. Este control ha conseguido que este área sea una de las mejores áreas del INSALUD en lo que se refiere a ajuste presupuestario farmacéutico.

Este estudio pretende describir, dentro de nuestro ámbito de actuación con un estrecho seguimiento del gasto farmacéutico, cómo manejan los MAPs la hipercolesterolemia en los ancianos entre 65 y 75 años y su adecuación a las recomendaciones del National Cholesterol Education Program $(\mathrm{NCEP} \text { II })^{18}$.

\section{MATERIAL Y MÉTODO}

Estudio observacional transversal realizado en 1998 en el Área 10 de Madrid (INSALUD), que incluye los municipios de Getafe, Pinto, Parla y Griñón, con una población aproximada de 220.000 habitantes.

\section{Población de estudio}

- Criterio de inclusión: Médico de Atención Primaria (MAPs) perteneciente al Área 10 INSALUD, Madrid. Las variables de respuesta fueron 
evaluadas a través del manejo de la dislipemia en sus respectivos pacientes entre 65 y 75 años con hipercolesterolemia de más de un año de evolución.

-Criterios de exclusión:

- MAPs que rechacen el consentimiento informado.

- EAPs cuyas características diferenciales con el resto de EAPs del Área 10 sean muy evidentes (consultorios aislados de zonas rurales, o EAPs con menos de 5 facultativos).

- Pedíatras.

- Los investigadores que participaron en este estudio.

\section{Muestra}

El tamaño muestral se calculó con una estimación del nivel de cumplimiento del $45 \%$, una precisión de $\pm 0,03$ y un nivel de confianza (1- $\alpha$ ) del $95 \%$. Se realizó un muestreo bietápico por conglomerados. Excluimos del muestreo inicial a 2 equipos y a sus facultativos por tener características diferenciales notables con el resto de los EAPs del Área, uno por ser una zona rural y otro por sólo tener tres facultativos. Por muestreo aleatorio con probabilidades proporcionales al número de MAPs de las unidades primarias (16 EAPs del Área 10 con 125 MAPs en plantilla), se eligieron a 4 EAPs (25\%) con 38 MAPs. Dada una población finita de 841 pacientes con hipercolesterolemia, y corrigiendo el tamaño de la muestra para poblaciones finitas, se precisaba una muestra mínima necesaria de 468 sujetos de estudio. Mediante muestreo aleatorio simple entre los 841 sujetos elegibles de los EAPs seleccionados, se obtuvo una muestra de 551 pacientes entre 65 y 75 años con hipercolesterolemia de más de un año de evolución.

\section{Criterio de evaluación y factor de estudio}

Se eligió el criterio de evaluación de las recomendaciones del National Cholesterol Education Program (NCEP II) ${ }^{18}$, por ser las más avaladas entre la población médica.

"Los niveles iniciales de colesterol-LDL que se consideran adecuados para indicar la farmacoterapia en la hipercolesterolemia son: $\geq 190 \mathrm{mg} / \mathrm{dl}$ (4,9 mmol/l) si el paciente es asintomático con $<2$ FRCIs; $\geq 160 \mathrm{mg} / \mathrm{dl}(4,14 \mathrm{mmol} / \mathrm{l})$ si tiene $\geq 2$ FRCIs; $y \geq 130 \mathrm{mg} / \mathrm{dl}(3,36 \mathrm{mmol} / \mathrm{l})$ si tiene CI u otra forma de EVA.

-Excepciones:

- Pacientes con historia de reacciones adversas a los agentes hipolipemiantes.
- Que no hayan venido a consulta en el año anterior.

- Pacientes con contraindicaciones en el uso de agentes hipolipemiantes (colelitiasis, hiperparatiroidismo, urolitiasis, hepatopatías, tests de función hepática alterado, miopatías, hemopatías).

- Pacientes terminales.

- La terapia farmacológica puede iniciarse si, tras la modificación de la dieta y de los estilos de vida durante 3-6 meses, no se han conseguido los niveles deseados."

Se cumple el criterio si el MAP utiliza o no fármacos hipolipemiantes ante la población asintomática con $\mathrm{LDL} \geq 190 \mathrm{mg} / \mathrm{dl}(4,9 \mathrm{mmol} / \mathrm{l})$ y $\sin \geq 2$ FRCIs ni CI/EVA.

Se consideran factores de riesgo de cardiopatía isquémica (FRCIs):

-Varones $\geq 45$ años.

-Mujeres $\geq 55$ años o menopausia prematura sin tratamiento estrogénico sustitutivo.

- Historia familiar de cardiopatía isquémica prematura (infarto agudo de miocardio, muerte repentina antes de los 55 años en padre o familiar varón de $1^{\text {er }}$ grado, o antes de los 65 años en madre o familiar mujer de $1^{\text {er }}$ grado). día.

- Tabaquismo: fumador de más de un cigarro al

- Hipertensión arterial confirmada $(\geq 140 / 90$ $\mathrm{mmHg}$ ), hipertensión arterial sistólica aislada, o uso de antihipertensivos.

-Diabetes mellitus: confirmación de glucemias en ayunas $\geq 126 \mathrm{mg} / \mathrm{dl}$ ( $7 \mathrm{mmol} / \mathrm{l})$ o clínica de diabetes mellitus con glucemia de $\geq 200 \mathrm{mg} / \mathrm{dl}$ (11,1 $\mathrm{mmol} / \mathrm{l})$.

- HDL <35 mg/dl (0,9 mmol/l).

- HDL $\geq 60 \mathrm{mg} / \mathrm{dl}(1,55 \mathrm{mmol} / \mathrm{l})$ es un factor de riesgo negativo por lo que en su caso hay que sumar los factores de riesgo positivos y restar el negativo.

Se considera cardiopatía isquémica o enferme dad vascular arterioesclerótica (CI/EVA):

- Cardiopatía isquémica (evidencia clínica o de laboratorio de IAM o isquemia miocárdica clínicamente significativa, historia de cirugía o angioplastia coronaria, o evidencia de arteriosclerosis coronaria).

- Enfermedad arterial periférica (aneurisma aórtica abdominal o clínica o evidencia angiográfica de isquemia en extremidades).

-Arterioesclerosis carotídea.

-Accidentes isquémicos cerebrovasculares.

Se consideró obesidad si el IMC $\geq 30$. Para hipertrofia ventricular izquierda fueron válidos tanto criterios ecocardiográficos como electrocardiográficos. 
En todo caso se aceptan las consideraciones diagnósticas que el médico haya definido para su paciente, pues son dichas consideraciones las que el médico emplea para el tratamiento de la hipercolesterolemia de su paciente. Las determinaciones cuantitativas de cada FRCI que se utilizaron para la evaluación fueron la última o la media de las últimas obtenidas en el último año. El laboratorio del Hospital Universitario de Getafe realizó las determinaciones de los EAPS evaluados.

\section{Fuentes y recogida de datos}

Para la identificación y descripción de la población de estudio, se utilizaron los registros de morbilidad de los Centros de Salud y el Sistema de Información de los Centros de Atención Primaria (SICAP). La población de estudio se eligió según el método de muestreo descrito anteriormente. Se utilizaron las historias clínicas de estos pacientes para el estudio del grado de cumplimiento de los criterios.

\section{Aspectos éticos}

Los médicos fueron informados de su participación y objetivos del estudio, características básicas del diseño, y de sus posibles consecuencias, y se obtuvo el consentimiento informado. Los aspectos éticos fueron supervisados por la Comisión de Formación e Investigación del Área 10, por la Comisión del Uso Racional del Medicamento, y por el Comité Ético de Investigación Clínica del Hospital Universitario de Getafe.

\section{Variables evaluadas}

-Variable principal de respuesta: el criterio de evaluación es una variable de respuesta cualitativa dicotómica ("cumple" o "no cumple") definida como cumplimiento o no de los criterios que se describen en el factor de estudio.

\section{-Variables secundarias de respuesta:}

- Variables descriptivas del MAP: edad, años de antigüedad en el INSALUD, propietario o interino, especialista en Medicina Familiar y Comunitaria o no.

- Variables descriptivas del paciente: edad, sexo, diabetes, hipertensión arterial, tabaquismo, hipertrofia ventricular izquierda, obesidad, historia familiar de cardiopatía isquémica prematura, colesterol total, colesterol LDL, colesterol HDL, triglicéridos, CI, EVA, $n^{\circ}$ de FRCIs (Tablas I, II y III).
- Variables de diagnóstico y tratamiento:

- Diagnósticos: < 2 FRCIs sin CI/EVA, $\geq 2$ FRCIs, CI/EVA (Tabla II).

- Tratamientos: dieta, fibratos, estatinas, resinas, combinaciones (Tabla IV).

\section{Análisis estadístico}

Los grupos de estudio se evaluaron comparando el porcentaje de cumplimiento de los criterios mediante la prueba Chi cuadrado de Pearson $\left(\chi^{2}\right)$. Esta prueba también se utilizó para evaluar las demás variables cualitativas. Las medias y desviaciones estándar de las variables cuantitativas se evaluaron mediante el test de la t de Student para muestras independientes y con el análisis de varianza. Todos los análisis estadísticos se realizaron asumiendo un nivel $\alpha$ del 0,05. La precisión de las estimaciones se calculó con un grado de confianza del $95 \%$. Los análisis estadísticos se realizaron con la ayuda de los paquetes estadísti$\cos$ SPSS $^{25}$ y Epi-Info ${ }^{26}$.

\begin{tabular}{|c|c|c|c|c|}
\hline \multicolumn{5}{|c|}{$\begin{array}{l}\text { CARACTERÍSTICAS DESCRIPTIVAS } \\
\text { DE LOS SUJETOS DE ESTUDIO }\end{array}$} \\
\hline № pacientes $=551$ & $n$ & $\%$ & LI* (IC 95\%) & LS"\# (IC 95\%) \\
\hline Varones & 185 & 33,58 & 29,67 & 37,31 \\
\hline Mujeres & 366 & 66,42 & 62,29 & 70,33 \\
\hline Diabéticos & 187 & 33,94 & 30,02 & 38,08 \\
\hline Hipertensos & 377 & 68,42 & 64,33 & 72,25 \\
\hline Tabaquismo & 55 & 9,98 & 7,67 & 12,87 \\
\hline$I M C>30$ & 352 & 63,88 & 59,70 & 67,87 \\
\hline HVI & 60 & 10,89 & 8,47 & 13,87 \\
\hline A.F.Cl & 52 & 9,44 & 7,19 & 12,27 \\
\hline $\mathrm{Cl}$ & 63 & 11,43 & 8,96 & 14,46 \\
\hline EVA $(\operatorname{Sin} \mathrm{Cl})$ & 65 & 11,80 & 9,28 & 14,86 \\
\hline $\mathrm{HDL}<35 \mathrm{mg} / \mathrm{dl}$ & 20 & 3,63 & 2,29 & 5,65 \\
\hline $\mathrm{HDL} \geq 60 \mathrm{mg} / \mathrm{dl}$ & 170 & 30,85 & 27,05 & 34,92 \\
\hline $0 \mathrm{FRCls}$ & 42 & 7,62 & 5,61 & 10,25 \\
\hline $1 \mathrm{FRCl}$ & 140 & 25,41 & 21,87 & 29,30 \\
\hline$\geq 2 \mathrm{FRCls}$ & 369 & 66,97 & 62,84 & 70,85 \\
\hline $2 \mathrm{FRCls}$ & 210 & 38,11 & 34,06 & 42,33 \\
\hline $3 \mathrm{FRCls}$ & 126 & 22,87 & 19,47 & 26,65 \\
\hline $4 \mathrm{FRCls}$ & 33 & 5,99 & 4,22 & 8,40 \\
\hline \multicolumn{5}{|c|}{$\begin{array}{l}\text { n: no de pacientes con matriz de fila. } \\
\text { \# LI: límite inferior del intervalo de confianza al } 95 \% . \\
\text { \#\# LS: límite superior del intervalo de confianza al 95\%. } \\
\text { IMC: índice de masa corporal. } \\
\text { HVI: hipertrofia ventricular izquierda. } \\
\text { A.F.Cl: antecedente familiar de cardiopatía isquémica prematura. } \\
\text { CI: cardiopatía isquémica. } \\
\text { EVA: enfermedad vascular arterioesclerótica. } \\
\text { FRCIs: factores de riesgo para cardiopatía isquémica. }\end{array}$} \\
\hline
\end{tabular}




\begin{tabular}{|c|c|c|c|c|}
\hline \multicolumn{5}{|c|}{$\begin{array}{l}\text { Tabla II } \\
\text { CO DE LA POBLACIÓN DE ESTUDIO }\end{array}$} \\
\hline № pacientes $=551$ & $n$ & $\%$ & LI* (IC 95\%) & $\mathrm{LS}^{\# \#(I C ~ 95 \%)}$ \\
\hline $\begin{array}{l}\text { Sin Cl o EVA } \\
\text { ni } \geq 2 \text { FRCls }\end{array}$ & 155 & 28,13 & 24,45 & 32,12 \\
\hline$\geq 2 \mathrm{FRCls}$ & 369 & 66,97 & 62,84 & 70,85 \\
\hline $\begin{array}{l}\geq 2 \mathrm{FRCls} \\
\text { Sin Cl o EVA }\end{array}$ & 285 & 51,72 & 47,46 & 55,96 \\
\hline $\begin{array}{l}\mathrm{Cl} \text { o EVA } \\
\mathrm{Sin} \geq 2 \mathrm{FRCls}\end{array}$ & 27 & 4,90 & 3,31 & 7,14 \\
\hline $\begin{array}{l}\text { Cl o EVA } \\
y \geq 2 \text { FRCls }\end{array}$ & 84 & 15,25 & 12,40 & 18,58 \\
\hline $\mathrm{Cl} \circ \mathrm{EVA}$ & 111 & 20,15 & 16,92 & 23,79 \\
\hline $\begin{array}{l}\mathrm{Cl} \text { o EVA } \\
0 \geq 2 \mathrm{FRCls}\end{array}$ & 396 & 71,87 & 67,88 & 75,55 \\
\hline \multicolumn{5}{|c|}{$\begin{array}{l}\text { n: no de pacientes con matriz de fila. } \\
\text { *LI: límite inferior del intervalo de confianza al } 95 \% \text {. } \\
\text { \# LS: límite superior del intervalo de confianza al } 95 \% \text {. } \\
\text { CI: cardiopatía isquémica. } \\
\text { EVA: enfermedad vascular arterioesclerótica. } \\
\text { FRCls: factores de riesgo para cardiopatía isquémica. }\end{array}$} \\
\hline
\end{tabular}

\section{RESULTADOS}

Todos los resultados entre corchetes muestran los límites inferior y superior del IC 95\% de la variable que se enuncie. La edad media de los MAPs analizados fue de 38,18 $(36,60 ; 39,76) \pm 4,96$ años con una antigüedad en el INSALUD de $4,89(4,22 ; 5,56) \pm$ 2,12 años. El 34,21\% $(20,14 ; 51,42)$ de los MAPs eran propietarios, y el $36,84 \%(22,29 ; 54,00)$ eran especialistas en Medicina Familiar y Comunitaria.

La población estudiada tenía una edad media de $69,86(69,61 ; 70,11) \pm 2,98$ años y habitaban en municipios de Parla y Getafe con una población de más de 70.000 habitantes cada uno. Los percentiles de edad (años) de la distribución muestral son: p5 $=66 ; \mathrm{p} 10=66 ; \mathrm{p} 25=67 ; \mathrm{p} 50=69 ; \mathrm{p} 75=72 ; \mathrm{p} 90$ $=74 ; \mathrm{p} 95=75$.

La descripción de las características descriptivas de los pacientes se expone en la tabla 1. Sólo el 28,13\% de los pacientes no tenían <2 FRCIs ni CI u otra EVA, dos terceras parte de la población estudiada padecía $\geq 2$ FRCIs, y una quinta parte tenía CI/EVA (Tabla II). El perfil lipídico de los pacientes se describe exhaustivamente en la tabla III. Mientras que el uso exclusivo de la dieta para el tratamiento de la hipercolesterolemia se realiza mayoritariamente en las dos terceras partes de los pacientes, en cambio sólo se usan estatinas en una quinta parte de los pacientes, y los fibratos en una octava parte (Tabla IV).

El nivel de cumplimiento de los criterios de adecuado manejo de la hipercolesterolemia no llega a la mitad $(47,91 \%)$ de los pacientes estudiados. Cuando los pacientes no tienen $\geq 2$ FRCIs, CI ni otra EVA, el nivel de cumplimiento mejora y supera las dos terceras partes de ellos. Sin embargo y paradójicamente, el cumplimiento disminuye al $41 \%$ si tienen $\geq 2$ FRCIs, y no llega al $37 \%$ si tienen CI/EVA (Tabla V). Cuando la dislipemia se maneja exclusivamente con dieta, el nivel de cumplimento de adecuado manejo de la dislipemia llega al $31 \%$. Si se utilizan además los fibratos, el nivel de cumplimento de los criterios de la NCEP II prácticamente se duplica, y si se usan las estatinas, el cumplimento casi se triplica (Tabla VI).

\begin{tabular}{|c|c|c|c|c|c|c|c|c|c|}
\hline \multirow[b]{2}{*}{ № pacientes 551} & \multirow[b]{2}{*}{ Mín. } & \multirow[b]{2}{*}{ p25 } & \multirow[b]{2}{*}{ p50 } & \multirow[b]{2}{*}{ p75 } & \multirow[b]{2}{*}{ Máx. } & \multirow[b]{2}{*}{ Media } & \multicolumn{3}{|c|}{ Media } \\
\hline & & & & & & & $\mathrm{DE}$ & $\mathrm{LI}^{\#}(\mathrm{IC}$ 95\%) & $\mathrm{LS}^{\# \#}(\mathrm{IC} 95 \%)$ \\
\hline Colest. Total (mg/ dl*) & 175 & 235 & 255 & 279 & 377 & 258,35 & $\pm 33,50$ & 255,55 & 261,15 \\
\hline Colesterol HDL (mg/ dl*) & 27 & 46 & 53 & 62 & 108 & 55,08 & $\pm 13,61$ & 53,94 & 56,22 \\
\hline Colesterol LDL (mg/ dl*) & 88 & 148 & 169 & 191 & 299 & 170,88 & $\pm 33,65$ & 168,07 & 173,69 \\
\hline Índice aterogénic $0 * *$ & 2,3 & 4,1 & 4,9 & 5,6 & 12 & 4,94 & $\pm 01,26$ & 4,83 & 5,05 \\
\hline Triglicéridos (mg/ $\mathrm{dl} * * *$ ) & 48 & 103 & 140 & 195 & 667 & 156,90 & $\pm 75,30$ & 150,61 & 163,19 \\
\hline
\end{tabular}

* Factor de conversión mg/ dl a mmol/ I de colesterol: multiplicar por 0,0258.

**índice aterogénico: Col. Total/ Col. HDL.

***Factor de conversión $\mathrm{mg} / \mathrm{dl} \mathrm{a} \mathrm{mmol} /$ I de triglicéridos: multiplicar por 0,0113.

DE: desviación estándar.

" $L$ I: límite inferior del intervalo de confianza al 95\%.

"\#LS: límite superior del intervalo de confianza al 95\%. 
Tabla IV

TRATAMIENTO DE LA HIPERCOLESTEROLEMIA

\begin{tabular}{lrrrc} 
№pacientes = 551 & \multicolumn{1}{c}{$n$} & \multicolumn{1}{c}{$\%$} & Ll $^{*}$ (IC 95\%) & LS\#\# (IC 95\%) \\
\hline Dieta & 354 & 64,25 & 60,07 & 68,23 \\
Fibratos & 67 & 12,16 & 9,61 & 15,25 \\
Resinas & 13 & 2,36 & 1,32 & 4,11 \\
Estatinas & 115 & 20,87 & 17,60 & 24,56 \\
Combinaciones & 2 & 0,36 & 0,06 & 1,45 \\
\hline
\end{tabular}

\# LI: límite inferior del intervalo de confianza al 95\%.

\#\# LS: límite superior del intervalo de confianza al $95 \%$.

$n: n o$ de pacientes en tratamiento con (matriz de fila).

\begin{tabular}{|c|c|c|c|c|}
\hline \multicolumn{5}{|c|}{ NIVEL DE CUMPLIMIENTO CONTROLADO POR DIAGNÓSTICOS } \\
\hline Manejo adecuado & $n / N$ & $\%$ & LI\# (IC 95 & S\#\# (IC 95\%) \\
\hline $\begin{array}{l}\text { Sin Cl o EVA } \\
\mathrm{ni} \geq 2 \mathrm{FRCls}\end{array}$ & $106 / 155$ & 68,39 & 60,36 & 75,48 \\
\hline$\geq 2 \mathrm{FRCls}$ & $117 / 285$ & 41,05 & 35,33 & 47,02 \\
\hline $\mathrm{Cl} O \mathrm{EVA}$ & $41 / 111$ & 36,94 & 28,12 & 46,67 \\
\hline Global & $264 / 551$ & 47,91 & 43,68 & 52,17 \\
\hline \multicolumn{5}{|c|}{$\begin{array}{l}\text { \# LI: límite inferior del intervalo de confianza al 95\%. } \\
\text { \#\# LS: límite superior del intervalo de confianza al 95\%. } \\
\text { n: no de pacientes con manejo adecuado. } \\
\text { N: no de pacientes con (matriz de fila). } \\
\text { Cl: cardiopatía isquémica. } \\
\text { EVA: enfermedad vascular arterioesclerótica. } \\
\text { FRCls: factores de riesgo para cardiopatía isquémica. }\end{array}$} \\
\hline
\end{tabular}

\section{Tabla VI}

NIVEL DE CUMPLIMIENTO CONTROLADO POR TRATAMIENTOS

\begin{tabular}{lllcl}
\hline Manejo adecuado & $\mathrm{n} / \mathrm{N}$ & $\%$ & Ll* (IC 95\%) & LS\# (IC 95\%) \\
\hline Dieta & $110 / 354$ & 31,07 & 26,34 & 36,22 \\
Fibratos & $41 / 67$ & 61,91 & 48,48 & 72,62 \\
Estatinas & $100 / 115$ & 86,96 & 79,09 & 92,27 \\
Resinas & $11 / 13$ & 84,62 & 53,66 & 97,29 \\
\hline Global & $264 / 551$ & 47,91 & 43,68 & 52,17
\end{tabular}

\# LI: límite inferior del intervalo de confianza al 95\%.

\#\# LS: límite superior del intervalo de confianza al $95 \%$.

$n:$ no de pacientes con manejo adecuado.

$\mathrm{N}$ : $\mathrm{n}$ - de pacientes en tratamiento con (matriz de fila).

\section{DISCUSIÓN}

Los estudios observacionales se encuentran, por lo general, con el inconveniente de que para sus estimaciones sean lo más precisas posibles deben utilizar una elevada población muestral, con lo que el coste económico y de trabajo llega en ocasiones a ser prohibitivo. Para conseguir nuestro objetivo realizamos un muestreo bietápico por conglomerados para reducir el coste y dar viabilidad al proyecto, por lo que estimamos que nuestros resultados se pueden inferenciar al Área 10. En cuanto a la posibilidad de que tenga validez externa a otras áreas, habría que demostrar que nuestra área no tenga especiales características diferenciales con el resto del INSALUD.

La edad del paciente superior a los 65 años, o incluso pertenecer al estrato de población más anciana de mayores de 75 años, no debe constituir una contraindicación para el tratamiento ${ }^{8}$. Sin embargo, no hay un común denominador en el manejo de la hipercolesterolemia ${ }^{9-12}$ de la población anciana. Apoyándonos en la bibliografía ${ }^{13-23}$, decidimos que los criterios de buen manejo de las dislipemias en esta población debían ser claros e inequívocos, y las recomendaciones de la NCEP II lo eran. En este estudio, el 71,87\% de la población tiene $\geq 2$ FRCIs o CI/EVA, un porcentaje demasiado elevado para dejar esta población de riesgo sin la adecuada atención médica. Los médicos que atendían a sus pacientes sólo con dieta, alcanzaban un bajo nivel (30\%) de cumplimiento de los criterios del correcto manejo de las dislipemias. Si la dislipemia se manejaba con fibratos prácticamente se duplicaba, y si lo hacían con estatinas, casi se triplicaba. La hipercolesterolemia se maneja adecuadamente en casi el $48 \%$ de la población estudiada. Pero si la analizamos detalladamente, vemos que cuanto mayor es el riesgo cardiovascular del paciente, peor es el nivel de adecuación del manejo de la hipercolesterolemia a los criterios de la NCEP II. Si el paciente no padece CI/EVA o no tiene $\geq 2$ FRCIs, el cumplimiento podemos decir que es aceptable llegando al 68\%. En este caso, el debido cumplimiento prácticamente no exige tratamiento farmacológico y por tanto gasto farmacéutico. Sin embargo, según vamos incrementando el riesgo cardiovascular y siendo más exigible el tratamiento con fármacos hipolipemiantes, va disminuyendo el nivel de cumplimiento, de forma que si los pacientes tienen $\geq 2$ FRCIs, el cumplimiento disminuye al $41 \%$, y paradójicamente, cuando los pacientes ya han sufrido CI/EVA, aún disminuye más hasta no llegar al $37 \%$.

La respuesta al por qué esto ocurre debe ser demasiado compleja para contestarla aquí, y habría que argumentarla con más trabajos de investiga- 
ción en los que se incluyan el análisis de factores de muy diversa índole que puedan estar implicados entre los que quizás se encontrarían los económicos, la estrecha supervisión sobre los médicos, los indicadores de calidad de prescripción farmacológica, la educación médica continuada, o el ajuste presupuestario farmacéutico.

En una reciente publicación ${ }^{27}$, se analizaron en 4 áreas de salud de Madrid, incluida la nuestra, los indicadores de calidad de prescripción de hipolipemiantes en recetas de pensionistas (mayores o no de 65 años), mostrando que existía una prescripción inadecuada en el 68,13\% (684/1004) de los pacientes, siendo del $64,83 \%$ en pacientes entre 65 y 74 años (282/435), y del 87,63\% (170/ 194) en los mayores de 74 años. Estimamos que tanto los resultados de este estudio como los del nuestro, muestran una elevada prescripción inadecuada, y deben hacer reconsiderar a los médicos que se atienda correctamente a esta población, pues al mejorar la indicación de los hipolipemiantes, se adecua el gasto farmacéutico a las necesidades reales de la población.

\section{CORRESPONDENCIA:}

A. Ruiz García

EAP Ciudades

C/ Palestina s/n

28903 Getafe

Madrid

Tlfno: 916962358

Fax: 916960103

e-mail: antonioruiz@nacom.es

\section{Bibliografía}

1. Sytkowski PA, Kannel WB, D'Agostino RB. Changes in risk factors and the decline in mortality from cardiovascular disease. The Framingham Heart Study. N Engl J Med 1990; 322: $1635-41$.

2. Scandinavian Simvastatin Survival Study Group. Ensayo aleatorizado sobre reducción del colesterol en 4.444 pacientes afectados de cardiopatía coronaria: el Scandinavian Simvastatin Survival Study (4S). The Lancet (ed Esp.) 1995; 26 (3): $159-66$

3. Stampfer MJ, Sacks FM, Salvini S, Willett WC, Hennekens $\mathrm{CH}$. A prospective study of cholesterol, apolipoproteins and the risk of myocardial infartion. N Engl J Med 1991; 325: 373-81.

4. Manson JE, Gaziano JM, Ridker PM, Hennekens CH. Myocardial infartion: epidemiologic overview. En: Manson JE, Ridker PM, Gaziano JM, Hennekens CH, editores. Prevention of myocardial infartion. New York: Oxford University Press, 1996; 3-31.

5. National Center for Health Statistic Heart, Lung and Blood Institute Collaborative Lipid Group. Trends in serum cholesterol level among US adults aged 20 to 74 years: data from the National Health and Nutrition Examination Survey, 1960 to 1980 . JAMA 1987; 257: 937-42.

6. Ministerio de Sanidad y Consumo. Estudio de los factores de riesgo cardiovascular. Madrid: MSC, 1993.

7. Puska P. Comprehensive cardiovascular community control programs in Europe. WHO Euro Reports and Study 106. Copenhague: WHO, 1988.

8. Arnalich Fernández F. Tratamiento hipolipemiante: uso según factores de riesgo vascular. Inf Ter Sist Nac Salud 1996; 20: 1-6.

9. Villalbí JR, Martínez Martínez R. Tratar o no tratar la hipercolesterolemia. FMC 1994; 1: 425-39.

10. Martínez Martínez R, Martín P, Ciurana R, Martí M, Nubiola AR. Protocol de diagnòstic i tractament de les hiperlipè- mies 1991. Barcelona: Unitat Docent de Medicina Familiar i Comunitària de Barcelona, 1991.

11. Ciurana R. Tratamiento de la hipercolesterolemia en individuos de alto riesgo, en mujeres y en ancianos. Atención Primaria 1992; 9: 64-9.

12. Hulley SB, Newman TB. Cholesterol in the Elderly. Is it Important? JAMA. 1994; 272: 1372-4.

13. Carmena R, Ros E, Gómez-Gerique JA, Masana L, Ascaso JF. Documento Oficial de la Sociedad Española de arteriosclerosis. Recomendaciones para la prevención de la arteriosclerosis en España. Clin Invest Arteriosclerosis 1989; 1: 1-9.

14. Consenso para el control de la colesterolemia en España. Clin Invest Arteriosclerosis 1989; 1: 55-61.

15. Mata P, de Oya M, Pérez-Jiménez F, Ros Rahola E. Dieta y enfermedades cardiovasculares: recomendaciones de la Sociedad Española de Arteriosclerosis. Clin Invest Arteriosclerosis 1994; 6: 43-61.

16. Sociedad Española de Arteriosclerosis, Sociedad Española de Medicina Interna y Liga de la Lucha contra la Hipertensión Arterial. Recomendaciones para la prevención primaria de la enfermedad cardiovascular. Clin Invest Arteriosclerosis 1994; 6: 62-102.

17. Sociedad Española de Arteriosclerosis y Sociedad Española de Cardiología. Evidencias clínico experimentales y recomendaciones para el tratamiento de la hiperlipidemia en pacientes con cardiopatía isquémica. Clin Invest Arteriosclerosis 1994; 6: 103-11.

18. Expert Panel on Detection, Evaluation and Treatment of High Blood Cholesterol in Adults. Resumen del Segundo Informe del National Cholesterol Education Program (NCEP) por el Comité de Expertos para la Detección, Evaluación y Tratamiento de la Hipercolesterolemia en Adultos (Adult Treatment Panel II). JAMA (ed. esp.) 1993; 2: 670-81.

19. Due Criteria section of AJHP. Criteria for use of hypolipidemic agents in adults. Am J Hosp Pharm 1994; 51: 2837-40. 
20. Havel RJ, Rapaport E. Management of primary hyperlipidemia. N Engl J Med 1995; 332: 1491-8.

21. Study Group, European Atherosclerosis Society. The recognition and management of hyperlipidaemia in adults: a policy statement of the European Atherosclerosis Society. Eur Heart J 1988; 9: 571-600.

22. Gotto Am, Phil D, Paoletti R. New clinical guidelines of the European Atherosclerosis Society: focus on global risk. Lipiddigest 1993; 4: 1-8.

23. Pyöräla K, De Backer G, Graham I, Poole-Wilson P, Wood $\mathrm{D}$, et al. Prevention of coronary heart disease in clinical practice. Recommendations of the Task Force of the European Society of Cardiology, European Atherosclerosis
Society and European Society of Hypertension. European Heart J 1994; 15: 1300-31.

24. Gerencia del Área 10 del INSALUD. Memoria 1998. Madrid, 1999; 137-9.

25. SPSS for Windows (Release 6.1) Marija Norusis. SPSS PC Inc. Chicago, 1995.

26. Epi-Info, version 6 (A Word-Processing, Database, and Statistics Program for Public Health on IBM;-compatible Microcomputers) Andrew G. Dean 1994.

27. Sanz Cuesta T, Escortell Mayor E, Fernández San Martín MI, López Bilbao C, Medina Bustillo B, Torres Bouza C, et al. Calidad del tratamiento farmacológico en pacientes con hiperlipemia de 4 áreas de salud. Aten Primaria 2000; 26 (6): 368-73. 